\title{
Fourier transform infrared spectroscopic study of
}

\author{
abhurite $\mathrm{Sn}_{21} \mathrm{O}_{6} \mathrm{Cl}_{16}(\mathrm{OH})_{14}$ \\ Samuel Jouen*, Benoit Lefez, Moulay T. Sougrati, Béatrice Hannoyer \\ Laboratoire d'Analyse Spectroscopique et de Traitement de Surface des Matériaux \\ Université de Rouen - Institut des Matériaux - B.P. 12 \\ 76801 Saint Etienne du Rouvray Cedex France
}

\author{
*corresponding author: \\ Tel/Fax: +33 (0)2.32.95.50.72 \\ E-mail: samuel.jouen@univ-rouen.fr
}




\begin{abstract}
:
Abhurite $\mathrm{Sn}_{21} \mathrm{O}_{6} \mathrm{Cl}_{16}(\mathrm{OH})_{14}$ is a tin oxy-hydroxychloride discovered in 1985 as a tin corrosion product formed after long immersion in sea water, has been synthesised and studied using FTIRAS analysis. The vibrational spectra obtained in transmission and reflectance modes of analysis are presented and compared to the vibrational spectra determined by Kramers Kronig analysis. Transversal and longitudinal (TO and LO) optical modes have been identified.
\end{abstract}

Keywords: Abhurite, tin oxy-hydroxychloride, FTIRAS, Kramers Kronig 


\section{Introduction}

The corrosion behaviour of tin in various environments has been reported in numerous works. Stannic and stannous oxide appear as the main corrosion products even if the formation of tin(II) sulphates and tin(II) chlorides has been also detected respectively in industrial and saline environments [1-3]. Among these tin corrosion products, the tin oxyhydroxychloride $\mathrm{Sn}_{21} \mathrm{O}_{6} \mathrm{Cl}_{16}(\mathrm{OH})_{14}$, called abhurite, was discovered for the first time in 1985 on the surface of tin ingots submerged during 100 years in the Red Sea after a shipwreck [4]. It was also detected recently on the surface of pewter artifacts remaining from the Queen Anne's Revenge wreck that sank near Beaufort, North Carolina in 1718 [5]. The stability field diagram for the $\mathrm{SnO}-\mathrm{HCl}-\mathrm{H}_{2} \mathrm{O}$ system have been established at $298.2 \mathrm{~K}$ by Edwards et al. and points out that in natural environments, high salinity and low $\mathrm{pH}$ are the conditions which favour the formation of abhurite [6].

The crystallographic data available in the literature data are sufficient to perform the identification of abhurite with X-ray diffraction (XRD), but is efficient only for well crystallised materials. IR spectrometry enables us to rapidly obtain structural information even in cases of inefficiency of XRD. This compound was not really investigated by means of Fourier Transform InfRared Reflection Absorption Spectroscopy (FTIRAS). Von Schnering presents a single infrared spectrum of abhurite obtained in transmission mode without the exact location of the absorption bands [7]. No data is available for specular reflectance spectroscopy analysis. The reflection mode of analysis is a non-destructive investigation way that is essential to perform the analysis of archaeological artefacts corrosion layers. It is well established that the absorption bands position in reflection mode of analysis can differ from the transmission ones. Few parameters as the infrared radiation incidence angle, the composition and the thickness of the corrosion layers, are determining in reflectance spectroscopy. 
Consequently, the purpose of this work is to give the useful data necessary to perform efficiently the characterisation of $\mathrm{Sn}_{21} \mathrm{O}_{6} \mathrm{Cl}_{16}(\mathrm{OH})_{14}$ by FTIRAS. The infrared spectra of synthesised abhurite are presented in transmission and specular reflection modes and the main absorption bands are indexed and assigned. Moreover, a numerical analysis of the spectra was also performed using the Kramers-Kronig analysis. The effect of the thickness layer on the transverse and longitudinal (LO-TO) optical modes was also studied.

\section{Experimental section}

\section{Sample preparation}

A potentiostatic method was carried out to prepared $\mathrm{Sn}_{21} \mathrm{O}_{6} \mathrm{Cl}_{16}(\mathrm{OH})_{14}$ reference sample using an EGG-PAR 273A potentio-galvanostat. A three-electrode cell was set up, where the working electrode was a tin plate $\left(98.8 \%\right.$ purity). The tin surface $\left(35 \mathrm{~cm}^{2}\right)$ was freshly prepared by a mechanical polishing with 1000 -grade SiC paper. The counter electrode was a circular Pt grating and the reference an $\mathrm{Ag} / \mathrm{AgCl}$ electrode. $\mathrm{A} \mathrm{NaCl}$ solution $2 \mathrm{M}$ with a $\mathrm{pH}$ value of 6.6 was used as electrolyte. The experiment was conducted at room temperature using a constant electric current of $0.1 \mathrm{~mA} \cdot \mathrm{cm}^{-2}$ for one hour. At the end of the experiment, the $\mathrm{pH}$ of the electrolyte had reached a $\mathrm{pH}$ value in the range 11-12 and a grey corrosion layer had recovered the tin plate surface. This corrosion layer was removed mechanically from the surface and the resulting powder characterised by X-ray diffraction (fig.1) and Mössbauer spectroscopy was found to be the $\mathrm{Sn}_{21} \mathrm{O}_{6} \mathrm{Cl}_{16}(\mathrm{OH})_{14}$ crystallised phase only [8].

\section{Investigation methods}

XRD analysis was carried out with a Brucker D8 equipment using the Co-K $\alpha$ radiation $(\lambda=1.78897 \mathrm{~nm})$. 
FTIRAS spectra as function of the vibrational wavenumbers were collected with a Nicolet 710 instrument accumulating 128 interferograms in the energy range $4000-250 \mathrm{~cm}^{-1}$ at a resolution of $4 \mathrm{~cm}^{-1}$. In transmission mode, the sample analysed is a pellet containing $1 \mathrm{mg}$ of powder mixed and pressed with $300 \mathrm{mg}$ of $\mathrm{KBr}$. In specular reflection technique, two auxiliary optical instruments were used to provide a single reflection at $16^{\circ}$ and $80^{\circ}$ angles of incidence with respect to the perpendicular to the sample surface. The optical constants $n$ and $\mathrm{k}$ of the compound have been calculated using the Kramers-Kronig transformation.

\section{$\underline{\text { Results and discussion }}$}

FTIR is very useful to perform the characterization of corroded surface as it was demonstrated in previous works [9]. Contrary to X-ray analysis, both crystalline and amorphous corrosion products can be investigated. In the transmission mode of analysis, corrosion products have to be removed mechanically from the corroded surface whereas in specular reflection mode, the surface is directly investigated, the latter configuration being preferred for archaeological artifacts studies since it is non-destructive.

\section{Infrared spectra and optical parameters}

Transmission spectrum of $\mathrm{Sn}_{21} \mathrm{O}_{6} \mathrm{Cl}_{16}(\mathrm{OH})_{14}$ obtained in the range [3800-250] $\mathrm{cm}^{-1}$ is presented on Fig. 2. The exact location of each absorption band is given in the table 1 with tentative assignments based on literature works [10-11]. The spectrum is characterised by strong $\mathrm{OH}$ absorption bands in the region [3600-3200] $\mathrm{cm}^{-1}$ depending on the degree of hydrogen bonding. The peaks at 3564 and $3407 \mathrm{~cm}^{-1}$ are probably due to the non- or low hydrogen bonded $\mathrm{OH}$ absorption while the band at $3296 \mathrm{~cm}^{-1}$ can be assigned to $\mathrm{OH}$ groups involved in strong hydrogen bonds. The band located at $1621 \mathrm{~cm}^{-1}$ is characteristic of the $\mathrm{OH}$ bending mode in water. The few strong absorption bands located in the mid-infrared (1000- 
$300 \mathrm{~cm}^{-1}$ ) spectral region are in the absorption domain of fundamental vibrations of metallic ions of crystal lattice and can therefore be assigned to the $\mathrm{Sn}-\mathrm{O}$ and $\mathrm{Sn}-\mathrm{Cl}$ stretching modes.

Numerical analysis of reflectivity spectra using the Kramers Kronig method permits the determination of the refractive index $n$ and the extinction coefficient $k$ of abhurite. Measurements of the IR reflectance at nearly normal incidence have been made on pressed pellets composed of abhurite powder. The pellets, obtained by compression of the powder under vacuum with 10 tons by $\mathrm{cm}^{2}$, had a high-quality reflecting surface, with very low values of the roughness coefficient for IR optics. The spectrum acquired with this pellet represents the reflectivity $R$ as a function of the wavenumber $\omega$. Providing the measurement is done at near-normal reflectance, one may use the Kramers-Kronig relation to calculate the phase shift $\theta$ [12]. Knowing $R$ and $\theta$, the refractive index and the extinction coefficient can be calculated from the following equations [13-14]:

$$
\begin{gathered}
\mathrm{n}(\omega)=(1-\mathrm{R}) /\left(1+\mathrm{R}-2 \mathrm{R}^{1 / 2} \cos \theta\right) \\
\mathrm{k}(\omega)=(-2 \mathrm{R} \sin \theta) /\left(1+\mathrm{R}-2 \mathrm{R}^{1 / 2} \cos \theta\right)
\end{gathered}
$$

Fig. 3 shows the $n$ and $k$ values versus the wavenumber. Knowledge of the refractive index and extinction coefficient permits the determination of the complex dielectric function $\varepsilon(\omega)=\varepsilon_{1}(\omega)+i \varepsilon_{2}(\omega)$, using the relations:

$$
\begin{aligned}
& \varepsilon_{1}(\omega)=\mathrm{n}^{2}-\mathrm{k}^{2} \\
& \varepsilon_{2}(\omega)=2 \mathrm{nk}
\end{aligned}
$$

The real $\left(\varepsilon_{1}\right)$ and imaginary $\left(\varepsilon_{2}\right)$ parts of the complex dielectric function are plotted versus the vibrational frequency on fig. 4. Knowledge of this complex dielectric function, or of the complex index of refraction $\mathrm{N}(\omega)=\mathrm{n}(\omega)+\mathrm{ik}(\omega)$, is needed in a wide variety of applications such as the interpretation of spectral ellipsometry or reflection absorption infrared spectra. 


\section{Calculation of FTIRAS spectra}

From the dielectric parameters $\left(\varepsilon_{1}, \varepsilon_{2}\right)$, the reflection spectra can be calculated using the well-known expressions of specular reflectance of a thin dielectric film on an infinitely thick metallic substrate [15-16].

The peak maxima of the complex function imaginary part $\operatorname{Im}(\varepsilon)=\varepsilon_{2}$ and $\operatorname{Im}(-$ $1 / \varepsilon)=\varepsilon_{2} /\left(\varepsilon_{1}^{2}+\varepsilon_{2}^{2}\right)$ are associated with the presence of the transverse optical (TO) and the longitudinal optical (LO) modes, respectively [17]. Fig. 5 shows $\operatorname{Im}(\varepsilon)$ and $\operatorname{Im}(1 / \varepsilon)$ graphs as function of the wavenumber in the range $\left[550-300 \mathrm{~cm}^{-1}\right]$. Table 2 presents the LO and TO optical modes of abhurite thus determined.

The complex expression of the specular part of the reflectance has been calculated for the two $16^{\circ}$ and $80^{\circ}$ angles of incidence usually used in the specular reflection mode of analysis [18]. The specular reflection spectra calculated for a $0.25,0.5,0.75,1$ and $2 \mu \mathrm{m}$ thickness layer of abhurite on a tin substrate at incidence $16^{\circ}$ and $80^{\circ}$ are presented in the range $\left[1100-300 \mathrm{~cm}^{-1}\right]$ in fig $6 \mathrm{a}$ and $6 \mathrm{~b}$.

For a thin abhurite layer, the spectra exhibit the LO optical modes only at 497, 443, and $381 \mathrm{~cm}^{-1}$ while the TO optical modes located at 477, 427, $369 \mathrm{~cm}^{-1}$ appear to the detriment of LO modes with increasing thickness. The LO-TO splitting of the lower energy mode $\left(2 \mathrm{~cm}^{-1}\right)$ is not enough significant to note an evolution. Regarding to the bands due to hydroxyl $\mathrm{OH}$ deformations, located at 966 and $923 \mathrm{~cm}^{-1}$ in the transmission spectrum, the positions obtained by means of the calculated spectra are a little different and the intensities remain smaller. The positions are 972 and $930 \mathrm{~cm}^{-1}$ at incidence $80^{\circ}$ and stay roughly similar with various thicknesses while the position vary from 971 and $930 \mathrm{~cm}^{-1}$ to 966 and $924 \mathrm{~cm}^{-1}$ with increasing thickness for the incidence $16^{\circ}$ as it is illustrated in fig. 7 .

In order to compare the simulated and experimental spectra, the fig. 8 presents an experimental spectrum obtained at $16^{\circ}$ of incidence on tin coupons covered with a layer of 
pure synthesised abhurite $\mathrm{Sn}_{21} \mathrm{O}_{6} \mathrm{Cl}_{16}(\mathrm{OH})_{14}$. One can note that the experimental spectrum is well in accordance with calculated spectra determined at $16^{\circ}$ of incidence. There are some discrepancies for the intensity of the bands in the energy range higher than $500 \mathrm{~cm}^{-1}$. Such changes in the relative intensities have already been observed [19]. The band located near 630 $\mathrm{cm}^{-1}$ was assigned to a stretching mode, but we cannot exclude the addition of other vibration modes. On this assumption, the extinction coefficient extracted from the Kramers Kronig analysis could be underevaluated. Regarding to the location of the absorption bands, it is quite similar to the calculated spectrum obtained for a layer with thickness of about $2 \mu \mathrm{m}$. 


\section{Conclusion}

Abhurite $\mathrm{Sn}_{21} \mathrm{O}_{6} \mathrm{Cl}_{16}(\mathrm{OH})_{14}$ has been synthesised and studied in details by FTIRAS analysis. The positions of the absorption bands were determined from experimental spectra in transmission and reflection mode of analysis. Using the Kramer Kronig analysis, the refractive index $\mathrm{n}$, the extinction coefficient $\mathrm{k}$ and the dielectric parameters $\varepsilon_{1}$ and $\varepsilon_{2}$ of abhurite were determined and used to identify the LO and TO optical modes.

The displacement of the absorption bands and the appearance of LO and TO optical modes with the thickness of the abhurite layer on reflection spectra at $16^{\circ}$ and $80^{\circ}$ angles of incidence were also examined. 


\section{References}

[1] V.S. Peters, R. Paintaske, G. Hecht, Korrosion, 18 (1987) 39.

[2] T. Biestek, M. Drys, Powloki Ochr. 1 (1981) 5.

[3] Jouen S, Thesis, Université de Rouen (2000)

[4] J. J. Matzko, H. T. Evans, M. E. Mrose, P. Aruscavage, Can. Mineral. 23 (1985) 233.

[5] S. E. Dunkle, J. R. Craig, J. D. Rimstidt, Can. Mineral. 41 (2003) 659.

[6] R. Edwards, R. D. Gillard, P. A. Williams, Mineral. Mag. 56 (1992) 221.

[7] H. G. Von Schnering, R. Nesper, H. Pelshenke, Z. Naturforsch. 36b (1981) 1551.

[8] M. T. Sougrati, S. Jouen, B. Hannoyer, Hyperfine Interact. 167 (2006) 815.

[9] B. Lefez, S. Jouen, J. Kasperek, B. Hannoyer, Appl. Optics, 55 (2001) 935.

[10] W. Martens, R. L. Frost, P.A.Williams, N. Jb. Miner. Abh, 178 (2003) 197.

[11] J. Y. Malvault, J. Lopitaux, D. Delahaye, M. Lenglet, J. Appl. Electrochem. 25 (1995) 841.

[12] T. Anki, B. Lefez, Appl. Optics, 35 (1996) 1399.

[13] M. Roesseler, Br. J. Appl. Phys. 16 (1965) 1119.

[14] J. Himmrich, G. Schneider, J. Zwinscher, H. D. Lutz, Naturforsch Z. 46 (1991) 1095.

[15] G. C. Allen, G. A. Swallow. Oxid. Met. 17 (1982) 157.

[16] D. A. Renneke, D. W. Lynch, Phys. Rev. 138 (1965) A530.

[17] H. D. Lutz, B. Müller, H. J. Steiner, J. Sol. Stat. Chem. 90 (1991) 54.

[18] B. Lefez, M. Lenglet, Surf. Interface Anal.22 (1994) 456.

[19] D. Persson, C. Leygraf, J. Electrochem. Soc. 142 (1995) 1459. 